Pacific Journal of Mathematic 


\title{
ON THE NUMBER OF BI-COLORED GRAPHS
}

\author{
FRANK HARARY
}

1. Introduction. This is an extension of papers $[2,3,4]$ whose notation and terminology will be used. The main result is a formulation of the generating function or counting polynomial of bicolored graphs, obtained by the enumeration methods of Pólya [6]. A modification of the method yields the number of balanced signed graphs, solving a problem proposed in [5]. In the process of enumerating bicolored graphs, we consider two binary operations on permutation groups called "cartesian product" and "exponentiation" which are abstractly but not permutationally equivalent to the direct product and Polya's "Gruppenkranz" [6], respectively.

A graph consists of a finite set of points together with a prescribed subset of the collection of all lines, i.e., unordered pairs of distinct points. Two points are adjacent if there is a line joining them. A graph is $k$-chromatic ${ }^{1}$ if each of the points can be assigned one of $k$ given colors so that any two adjacent points have different colors. A graph is $k$-colored if it is $k$-chromatic and its points are colored so that all $k$ colors are used. More precisely, a $k$-colored graph is a pair $(G, f)$ where $G$ is a graph and $f$ is a function from the set of points of $G$ onto the set of numbers $1,2, \cdots, k$ such that if $a$ and $b$ are adjacent points, then $f(a) \neq f(b)$. Two graphs are isomorphic if there exists a one-to-one adjacency preserving transformation between their sets of points. Two $k$-colored graphs are chromaticallg isomorphic if there is a color preserving isomorphism between them. Thus $\left(G_{1}, f_{1}\right)$ is chromatically isomorphic with $\left(G_{2}, f_{2}\right)$ if there is an isomorphism $\theta: G_{1} \rightarrow G_{2}$ and a permutation $\omega:\{1, \cdots, k\} \rightarrow\{1, \cdots, k\}$ such that $\omega\left(f_{1}(a)\right)=f_{2}(\theta(a))$ for every point $a$ in $G_{1}$. Let $g_{p, q}^{(k)}$ be the number of chromatically nonisomorphic $k$-colored graphs with $p$ points and $q$ lines, and let the corresponding generating function be

$$
g_{p}^{(k)}(x)=\sum_{q=0}^{p(p-1) / 2} g_{p, q}^{(k)} x^{q}
$$

We first derive the number of bicolored graphs, $k=2$, and then discuss the formula for $k=3$. The problem remains open for $k>2$.

Received November 4, 1957, and in revised forms March 28, 1958, and June 16, 1958. This work was supported by a grant from the National Science Foundation. The author is deeply grateful to the referee for making several insightful comments of clarification.

1 This definition is different from that of Dirac [1]. According to Dirac, a graph has chromatic number $k$ if it is $k$-chromatic but not $(k-1)$-chromatic as defined here. 
In precisely the form in which we require it, Pólya's enumeration theorem is reviewed briefly in $\$ 2$ of [2]. Therefore, we shall not repeat here the definitions leading up to it, but shall only restate the theorem itself.

PóLyA's THEOREM. The configuration counting series $F(x)$ is obtained by substituting the figure counting series $\varphi(x)$ into the cycle index $Z(\Gamma)$ of the configuration group $\Gamma$. Symbolically,

$$
F(x)=Z(\Gamma, \varphi(x)) \text {. }
$$

This theorem reduces the problem of finding the configuration counting series to the determination of the figure counting series and the cycle index of the configuration group.

2. Bicolored graphs; the cartesian product of permutation groups. Let $K_{n}$ be the complete graph of $n$ points, in which any two points are adjacent. Let $K_{m n}$ be the bicolored graph whose $m+n$ points are $a_{1}, a_{2}, \cdots, a_{m}, b_{1}, b_{2}, \cdots, b_{n}$ and whose $m n$ lines are all those of the form $a_{i} b_{j}$.

Clearly if a graph is $k$-colored then its point set is partitioned into $k$ disjoint non-empty subsets such that no two points in the same subset are adjacent. Hence a bicolored graph with $p$ points is a "line-subgraph" (as in [3]) or a spanning subgraph" of a graph $K_{m n}$ for which $m+n=p$. Let $g_{m n, p}$ be the number of chromatically nonisomorphic spanning subgraphs of $K_{m n}$ having $q$ lines, and let

$$
g_{m n}(x)=\sum_{q=0}^{m n} g_{m n, q} x^{q} .
$$

Then

$$
g_{p}^{(2)}(x)=\sum_{1 \leqq m \leqq n} g_{m n}(x) \text {, }
$$

where the sum is taken over all $m$ and $n$ such that $m+n=p$. Therefore in order to obtain a formula for the counting polynomial (4), it is sufficient to find that for (3). In this section, we find $g_{m n}(x)$ for the case $m \neq n$ using the "cartesian product" of two permutation groups. In the next section we see that this combinatorial technique is not valid for $m=n$ and formulate $g_{n n}(x)$ in terms of the "exponentiation" of the appropriate two permutation groups.

By Theorem 1 of [3], the counting polynomial $g_{m n}(x)$ for the

${ }^{2}$ A spanning subgraph of a graph $G$ is one whose set of points coincides with that of $G$. 
number of spanning subgraphs of $K_{m n}$ is obtained by substituting $1+x$ into the cycle index of the line-group ${ }^{3}$ of $K_{m n}$ :

$$
g_{m n}(x)=Z\left(\Gamma_{1}\left(K_{m n}\right), 1+x\right) .
$$

We note that this equation can also be obtained from the main result, equation (5), of [4]. For the subgraphs of $K_{m n}$ correspond to the different supergraphs of the union $K_{m} \cup K_{n}$ of two complete graphs on disjoint point sets. The derivation of $Z\left(\Gamma_{1}\left(K_{m n}\right)\right)$ for the case $m \neq n$ is parallel to and algebraically simpler than that of $Z\left(\Gamma_{1}\left(K_{p}\right)\right)$, which appears in $\S 3$ of [2]. Throughout the rest of this section we assume $m \neq n$.

The line group of $K_{m n}$ may be described as an appropriately formulated product of the symmetric groups $\mathbf{S}_{m}$ and $\mathbf{S}_{n}$. This product can be generally defined for any two permutation groups in the following way. Let $\mathbf{A}$ and $\mathbf{B}$ be any two permutation groups with object sets $X$ and $Y$, degrees $d$ and $e$ and orders $m$ and $n$ respectively. The cartesian product $\mathbf{A} \times \mathbf{B}$ of these two permutation groups has degree de and order $m n$. Its object set is the cartesian product of $X$ and $Y$ and each of its permutations $(\alpha, \beta)$ is the cartesian product of permutations $\alpha$ and $\beta$ from $\mathbf{A}$ and $\mathbf{B}$ defined by $(\alpha, \beta)(x, y)=(\alpha x, \beta y)$. As an abstract group, the cartesian product is isomorphic to the direct product AB, but they are not permutationally equivalent. For the degree of the direct product is $d+e$ since the group $\mathbf{A B}$ has $X \cup Y$ as its object set.

There is a precise method for finding the cycle index of a cartesian product in terms of the cycle indices of the two permutation groups. We first illustrate the method by finding $Z\left(\Gamma_{1}\left(K_{23}\right)\right)$. The line group of $K_{23}$ is the cartesian product of $\mathbf{S}_{2}$ and $\mathbf{S}_{3}$ which is a permutation group of degree 6 and order 12, written $\Gamma_{1}\left(K_{23}\right)=\mathbf{S}_{2} \times \mathbf{S}_{3}$. Let $a_{1}, a_{2}$ be the indeterminates occurring in $Z\left(\mathbf{S}_{2}\right)$ and $b_{1}, b_{2}, b_{3}$ be those in $Z\left(\mathbf{S}_{3}\right)$ so that

$$
Z\left(\mathbf{S}_{2}\right)=\frac{1}{2}\left(a_{1}^{2}+a_{2}\right) \text { and } Z\left(\mathbf{S}_{3}\right)=\frac{1}{6}\left(b_{1}^{3}+3 b_{1} b_{2}+2 b_{3}\right) .
$$

Then we write ${ }^{1}$

$$
\begin{aligned}
& Z\left(\Gamma_{1}\left(K_{23}\right)\right)=Z\left(\mathbf{S}_{2} \times \mathbf{S}_{3}\right)=Z\left(\mathbf{S}_{2}\right) \times Z\left(\mathbf{S}_{3}\right) \\
& =\frac{1}{12}\left(a_{1}^{2} \times b_{1}^{3}+3 a_{1}^{2} \times b_{1} b_{2}+2 a_{1}^{2} \times b_{3}+a_{2} \times b_{1}^{3}+3 a_{2} \times b_{1} b_{2}+2 a_{2} \times b_{3}\right),
\end{aligned}
$$

${ }_{3}$ The line group $\Gamma_{1}(G)$ of a graph $G$ is the collection of all permutations on the set of lines of $G$ consistent with the automorphism group $\Gamma(G)$ of $G$; see [3].

4 By the following formulas we mean that the cartesian product of two permutation groups can be exten sed to the cartesian product of their cycle indices in the indicated manner. 
and give each of these six terms in Table 1 , in which $c_{1}$ to $c_{6}$ denote the indeterminates in $Z\left(\Gamma_{1}\left(K_{23}\right)\right)$.

Table 1

\begin{tabular}{c|cccccc} 
Term of $Z\left(\mathbf{S}_{2} \times \mathbf{S}_{3}\right)$ & $a_{1}^{2} \times b_{1}^{3}$ & $a_{1}^{2} \times b_{1} b_{2}$ & $a_{1}^{2} \times b_{3}$ & $a_{2} \times b_{1}^{3}$ & $a_{2} \times b_{1} b_{2}$ & $a_{2} \times b_{3}$ \\
\hline Term of $Z\left(\Gamma_{1}\left(K_{23}\right)\right)$ & $c_{1}^{6}$ & $c_{1}^{2} c_{2}^{2}$ & $c_{3}^{2}$ & $c_{2}^{3}$ & $c_{2}^{3}$ & $c_{3}$
\end{tabular}

We illustrate Table 1 for the term $a_{2} \times b_{1} b_{2}$. Let the 2-cycle $\left(p_{1} p_{2}\right)$ stand for $a_{2}$ and the 1-cycle and 2-cycle $\left(q_{1}\right)\left(q_{2} q_{3}\right)$ for $b_{1} b_{2}$. The admissible lines of $K_{23}$ are only those of the form $p_{i} q_{j}$. The pair $p_{1} q_{1}$ is transformed into $p_{2} q_{1}$, and then back again to give the cycle of length 2 in the corresponding permutation of $\Gamma_{1}\left(K_{23}\right)$ of the form $\left(p_{1} q_{1} p_{2} q_{1}\right)$. Similarly the transpositions $\left(p_{1} q_{2} p_{2} q_{3}\right)$ and $\left(p_{1} q_{3} p_{2} q_{2}\right)$ are factors of this element of $\Gamma_{1}\left(K_{23}\right)$. Altogether there are three transpositions, so the corresponding term of $Z\left(\Gamma_{1}\left(K_{23}\right)\right)$ is $c_{2}^{3}$.

In general, we have

$$
a_{1}^{i} a_{2^{2}}^{i} \cdots a_{m}^{i} \times b_{1}^{j} b_{2}^{j} \cdots b_{n}^{j} n=\prod_{\alpha, \beta}\left(a_{\alpha^{\alpha}}^{i} \times b_{\beta}^{j} \beta\right)
$$

and

$$
a_{\alpha^{\alpha}}^{i} \times b_{\beta}^{j} \beta=c_{m(\alpha, \beta)}^{i \alpha^{j} \beta^{d(\alpha, \beta)}}
$$

where $d(\alpha, \beta)$ and $m(\alpha, \beta)$ are the greatest common divisor and least common multiple.

The cycle index of $\mathrm{S}_{p}$ is

$$
Z\left(\mathbf{S}_{p}\right)=\frac{1}{p !} \sum_{(j)} \frac{p !}{1^{j_{1} j_{1} ! \cdots p^{j} j_{p} !}} f_{1}^{{ }^{j}} \cdots f_{p}{ }^{j} p
$$

where the sum is taken over all partitions $(j)=\left(j_{1}, j_{2}, \cdots, j_{p}\right)$ of $p$ such that

$$
1 j_{1}+2 j_{2}+\cdots+p j_{p}=p .
$$

The last four equations together with

$$
Z\left(\Gamma_{1}\left(K_{m n}\right)\right)=Z\left(\mathbf{S}_{m}\right) \times Z\left(\mathbf{S}_{n}\right)
$$

provide a formula for $g_{m n}(x)$ when $m \neq n$.

We use Table 1 to illustrate equation (9) by finding $g_{23}(x)$. Here

$$
Z\left(\Gamma_{1}\left(K_{23}\right)\right)=\frac{1}{12}\left(c_{1}^{6}+3 c_{1}^{2} c_{2}^{2}+2 c_{3}^{2}+4 c_{2}^{3}+2 c_{6}\right),
$$

so that

$$
g_{23}(x)=1+x+3 x^{2}+3 x^{3}+3 x^{4}+x^{5}+x^{6} \text {. }
$$


The bicolored graphs with two points of one color and three points of the other color which correspond to the coefficients in the preceding counting polynomial are shown in Figure 1.

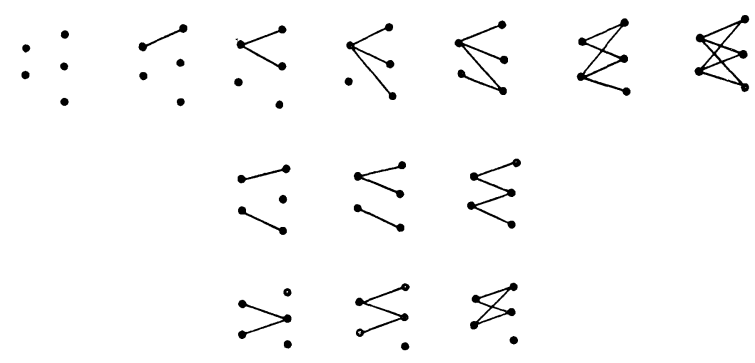

Fig. 1.

3. Bicolored graphs; exponentiation of permutation groups. We now turn to the enumeration of bicolored graphs for the case $m=n$. As in the preceding section, we again have equation (5) holding for this special case:

$$
g_{n n}(x)=Z\left(\Gamma_{1}\left(K_{n n}\right), 1+x\right) .
$$

However, it is not true that $\Gamma_{1}\left(K_{n n}\right)=\mathbf{S}_{n} \times \mathbf{S}_{n}$ since $\mathbf{S}_{n} \times \mathbf{S}_{n}$ is a proper subgroup of $\Gamma_{1}\left(K_{n n}\right)$. The remaining $(n !)^{2}$ permutations in $\Gamma_{1}\left(K_{n n}\right)$ are obtained on interchanging the two colors in accordance with the definition of chromatic isomorphism.

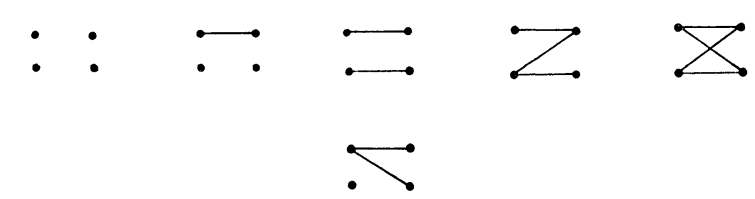

Fig. 2 .

For example, all the (chromatically nonisomorphic) bicolored graphs for $m=n=2$ are shown in Figure 2, so that $g_{22}(x)=1+x+2 x^{2}+x^{3}+x^{4}$. However, the formulas in the preceding section give

$$
Z\left(\mathbf{S}_{2} \times \mathbf{S}_{2}, 1+x\right)=1+x+3 x^{2}+x^{3}+x^{4},
$$

since the permutations in $\mathbf{S}_{2} \times \mathbf{S}_{2}$ distinguish between the two bicolored graphs in Figure 3, in which the color assigned to each point is indicated by one of the integers 1 or 2 .

With the appropriate definition of group exponentiation, we will express $\Gamma_{1}\left(K_{n n}\right)$ as $\mathbf{S}_{n}$ raised to the power $\mathbf{S}_{2}$. We first review the definition of the "composition" of two permutation groups (the "Gruppenkranz" of Pólya [6]). Let $\mathbf{A}$ and $\mathbf{B}$ be any two permutation groups as in the preceding section. Then using the notation of Pólya [6] the 
composition $\mathbf{A}[\mathbf{B}]$ of $\mathbf{A}$ with $\mathbf{B}$ has object set $X \times Y$ (as for the cartesian product). However, it is more convenient to regard the object set here as a $d$ by $e$ matrix $M=\left(x_{i j}\right)$. Then the elements of $\mathbf{A}[\mathbf{B}]$ are the permutations of the entries of $M$ constructed as follows. First permute the rows of $M$ in accordance with an element of $\mathbf{A}$. Then permute the column indices in each row separately using one element
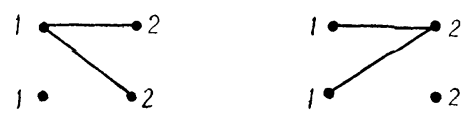

Fig. 3.

of $\mathbf{B}$ for each row, repetitions permitted. Hence the degree of $\mathbf{A}[\mathbf{B}]$ is de and the order is $m n^{a}$.

The exponentiation $\mathbf{B}^{\mathbf{A}}$ of $\mathbf{A}$ with $B$ is that permutation group whose object set is $Y^{x}$, the collection of all functions from $X$ into $Y$, and whose elements are constructed as follows. It is assumed that the objects $x_{1}, x_{2}, \cdots, x_{a}$ in $X$ are indexed. First permute the objects in $X$ in accordance with an element $\alpha$ of $\mathbf{A}$. Then for each object $x_{1}$ in $X$, permute the $e$ objects of $Y$ into which it can be mapped, using a permutation $\beta_{i}$ from $\mathbf{B}$. More precisely each selection of $\alpha \in \mathbf{A}$ and $\beta_{1}, \beta_{2}, \cdots, \beta_{a} \in \mathbf{B}$ (not necessarily distinct) determines a permutation of $Y^{x}$ which takes the function $f$ into the function $f^{*}$ defined by:

$$
f^{*}\left(x_{i}\right)=\beta_{i} f\left(\alpha x_{i}\right) \text { for all } x_{i} \in X ; \quad i=1,2, \cdots, d .
$$

It can easily be shown that distinct selections of $\alpha, \beta_{1}, \cdots, \beta_{a}$ lead to distinct permutations of $Y^{x}$ and that these permutations form a group.

The degree of $\mathbf{B}^{\mathbf{A}}$ is $e^{a}$ and the order is $m n^{d}$. It follows at once from their constructions that the group $\mathbf{B}^{\mathbf{A}}$ and $\mathbf{A}[\mathbf{B}]$ are isomorphic as abstract groups. But they are not equivalent as permutation groups since they have different degrees.

With this definition of exponentiation, it follows at once that the line group of $K_{n n}$ is given by

$$
\Gamma_{1}\left(K_{n n}\right)=\mathbf{S}_{p}^{\mathbf{S}_{2}} .
$$

Before calculating the cycle index of $\mathbf{S}_{n^{2}}^{\mathbf{S}_{2}}$, we illustrate for $n=2$ and 3 . Since $\mathbf{S}_{2}^{\mathbf{S}_{2}}=\mathbf{S}_{2}\left[\mathbf{S}_{2}\right]=\mathbf{D}_{4}$, the dihedral group of degree 4 and order 8 , its cycle index in terms of the indeterminates $c_{1}, c_{2}, c_{3}, c_{4}$ is given by

$$
Z\left(\mathbf{D}_{4}\right)=\frac{1}{8}\left(c_{1}^{4}+3 c_{2}^{2}+2 c_{1}^{2} c_{2}+2 c_{4}\right) \text {. }
$$

The correct polynomial $g_{22}(x)$ which verifies Figure 2 follows at once from this cycle index. 
For $n=3$, let the object set of $\Gamma_{1}\left(K_{33}\right)$ be denoted:

$$
X=\left\{11^{\prime}, 12^{\prime}, 13^{\prime}, 21^{\prime}, 22^{\prime}, 23^{\prime}, 31^{\prime}, 32^{\prime}, 33^{\prime}\right\} \text {. }
$$

Then $\Gamma_{1}\left(K_{33}\right)=\mathbf{S}_{3} \mathbf{S}_{2}$ contains the $(3 !)^{2}$ permutations in $\mathbf{S}_{3} \times \mathbf{S}_{3}$ and also the $(3 !)^{2}$ permutations obtained from these on multiplying each of them by the following reflection $\rho$ which interchanges primed and unprimed digits in the objects in $X$ :

$$
\rho=\left(11^{\prime}\right)\left(22^{\prime}\right)\left(33^{\prime}\right)\left(12^{\prime} 21^{\prime}\right)\left(23^{\prime} 32^{\prime}\right)\left(31^{\prime} 13^{\prime}\right) .
$$

Symbolically, we write

$$
\Gamma_{1}\left(K_{33}\right)=\mathbf{S}_{3}^{\mathbf{S}_{2}}=\left(\mathbf{S}_{3} \times \mathbf{S}_{3}\right) \cup \rho\left(\mathbf{S}_{3} \times \mathbf{S}_{3}\right) .
$$

Then

$$
Z\left(\mathbf{S}_{3} \times \mathbf{S}_{3}\right)=\frac{1}{(3 !)^{2}}\left(c_{1}^{9}+6 c_{1}^{3} c_{2}^{3}+8 c_{3}^{3}+9 c_{1} c_{2}^{4}+12 c_{3} c_{6}\right)
$$

and a straightforward calculation gives (using not quite proper notation since cycle index is defined for groups rather than cosets):

$$
Z\left(\rho\left(\mathbf{S}_{3} \times \mathbf{S}_{3}\right)\right)=\frac{1}{(3 !)^{2}}\left(6 c_{1}^{3} c_{2}^{3}+18 c_{1} c_{4}^{2}+12 c_{3} c_{6}\right) .
$$

Combining these, we have

$$
Z\left(\mathrm{~S}_{3}^{\mathrm{S}_{2}}\right)=\underset{2 \cdot(3 !)^{2}}{1}\left(c_{1}^{9}+12 c_{1}^{3} c_{2}^{3}+8 c_{3}^{3}+9 c_{1} c_{2}^{4}+18 c_{1} c_{4}^{2}+24 c_{3} c_{6}\right)
$$

from which one readily calculates using (10) and (5),

$$
g_{33}(x)=1+x+2 x^{2}+4 x^{3}+5 x^{1}+5 x^{5}+4 x^{6}+2 x^{7}+x^{8}+x^{9} \text {. }
$$

We now proceed to obtain a closed formula for $Z\left(\mathbf{S}_{n}^{\mathbf{S}_{2}}\right)$, thereby completing the explicit solution of the enumeration of bicolored graphs. The process of finding this cycle index is also analogous to the calculation of of $Z\left(\Gamma_{1}\left(K_{p}\right)\right)$ which appears in $\S 3$ of [2]. Clearly, the automorphism group of $K_{n n}$ is $\mathbf{S}_{2}\left[\mathbf{S}_{n}\right]$. For the complement $K_{n n}^{\prime}$ consists of two disjoint copies of $K_{n}$. By a result in Pólya [6], the cycle index of the composition of two permutation groups is the composition of their cycle indices. For example,

$$
Z\left(\mathbf{S}_{2}\left[\mathbf{S}_{3}\right]\right)=\frac{1}{2}\left[\left(\frac{1}{6}\left(a_{1}^{3}+3 a_{1} a_{2}+2 a_{3}\right)\right)^{2}+\frac{1}{6}\left(a_{2}^{3}+3 a_{2} a_{4}+2 a_{6}\right)\right]
$$

But we require here the cycle index of the line group of $K_{n n}$. There is a one-to-one correspondence between the terms of the cycle indices 
$Z\left(\mathbf{S}_{n} \mathbf{S}_{2}\right)$ and $Z\left(\mathbf{S}_{2}\left[\mathbf{S}_{n}\right]\right)$ with the same integral coefficients. Analogous to the terms in the above illustration of $Z\left(\mathbf{S}_{2}\left[\mathbf{S}_{3}\right]\right)$, let us write

$$
Z\left(\mathbf{S}_{2}\left[\mathbf{S}_{n}\right]\right)=\frac{1}{2}\left[\left(Z\left(\mathbf{S}_{n}\right)\right)^{2}+Z\left(\mathbf{S}_{n}(2)\right)\right]
$$

Thus $Z\left(\mathbf{S}_{n}(2)\right)$ is obtained from $Z\left(\mathbf{S}_{n}\right)$ on replacing each indeterminate $f_{k}$ by $f_{2 k}$.

The term of $Z\left(\mathrm{~S}_{n^{2}}\right)$ corresponding to the first term of the right hand member of equation (11) is $Z\left(\mathbf{S}_{n} \times \mathbf{S}_{n}\right)$. For bicolored graphs with $m \neq n$, this is the result of the preceding section. The term of $Z\left(\mathbf{S}_{n}^{S_{2}}\right)$ corresponding to the term $Z\left(\mathrm{~S}_{n}(2)\right)$ of (11) is derived as follows. Let the general term of $Z\left(\mathbf{S}_{n}(2)\right)$ be given by

$$
f_{2}^{j} f_{4}^{j} \cdots f_{2 n}^{j}{ }_{n}
$$

This term (12) occurs in the cycle index of the point group of $K_{n n}$. We require the corresponding term in the cycle index of the line group obtained by calculating the induced permutation on pairs of points from two disjoint sets. Let the letters $c_{i}$ be the indeterminates in the cycle index $Z\left(\mathbf{S}_{n}^{\mathbf{S}_{2}}\right)$. There are two contributions to $Z\left(\mathbf{S}_{n}^{\mathbf{S}_{2}}\right)$ arising from (12): those from each of the $n$ factors $f_{2 k}^{s_{k}}$ separately, and those from pairs of factors $f_{2 r}^{j} f_{2 s}^{j}, r \neq s$.

The contribution to the cycle index due to each factor in (12) is

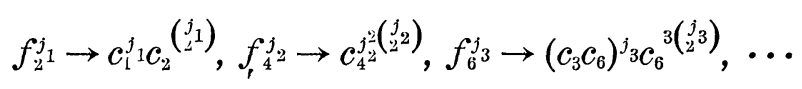

It is convenient to express the contribution of $f_{2 k}^{j}$ separately for $k$ even and $k$ odd:

$$
f_{2 k}^{j} k \rightarrow\left\{\begin{array}{l}
\left.\left(c_{2 k}{ }^{k / 2}\right)^{j_{k}} c_{2 k}{ }^{k}{ }_{2}^{j} k\right), k \text { even } \\
\left(c_{k} c_{2 k}{ }^{(k-1) / 2}\right)^{j_{k}} c_{2 k}{ }^{k\left({ }_{2}^{j} k\right)}, k \text { odd } .
\end{array}\right.
$$

Similarly, the contribution from pairs is given by

$$
f_{2 r}^{j_{r}} f_{2 s}^{j_{s}} \rightarrow c_{2 m(r, s)}^{j_{r}^{j} s_{s} d(r, s)}
$$$$
r<s,
$$

where $m(r, s)$ and $d(r, s)$ are the least common multiple and greatest common divisior respectively.

Collecting these observations, we find

$$
Z\left(\mathbf{S}_{n^{2}}\right)=\frac{1}{2}\left[Z\left(\mathbf{S}_{n} \times \mathbf{S}_{n}\right)+Z^{\prime}\right]
$$

where 


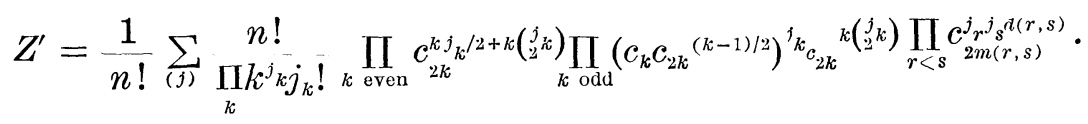

This formula for $Z\left(\mathbf{S}_{n^{2}}\right)$ together with equations (10) and (5) give the number of bicolored graphs for $m=n$. For $n=3$, this expression for $Z^{\prime}$ specializes to that for $Z\left(\rho\left(\mathbf{S}_{3} \times \mathbf{S}_{3}\right)\right)$ in the above example.

The only other known cycle index of the exponentiation of two permutation groups also involves complex combinatorial calculations and is worked out in Slepian [8]. Consider the counting polynomial $b_{n}(x)=$ $\sum b_{n m} x^{m}$, where $b_{n m}$ is the number of symmetry types of boolean functions of $n$ variables having $m$ nonzero terms when written in disjunctive normal form. Pólya [7] showed that

$$
b_{n}(x)=Z\left(\mathbf{Q}_{n}, 1+x\right),
$$

where $\mathbf{Q}_{n}$ is the automorphism group of the $n$-cube. It is easily seen that $\mathbf{Q}_{n}=\mathbf{S}_{2}^{\mathbf{S}_{n}}$ and in fact Pólya [7, footnote 7] comments that $\mathbf{Q}_{n}$ and $\mathbf{S}_{n}\left[\mathbf{S}_{2}\right]$ are isomorphic as abstract groups. Slepian [8] completed the enumeration problem for $b_{n}(x)$ by providing a calculus for an explicit formulation of $Z\left(\mathbf{S}_{2} \mathbf{S}_{1}\right)$, although using different terminology and notation.

It would be interesting to solve the general problem of obtaining an expression for $Z\left(\mathbf{B}^{\mathbf{A}}\right)$ in terms of $Z(\mathbf{A})$ and $Z(\mathbf{B})$. This would be analogous to equations (6) and (7) which give $Z(\mathbf{A} \times \mathbf{B})$ in terms of $Z(\mathbf{A})$ and $Z(\mathbf{B})$.

To summarize, the counting polynomial $g_{m n}(x)$ for bicolored graphs is given by

$$
g_{m n}(x)=\left\{\begin{array}{l}
Z\left(\mathbf{S}_{m} \times \mathbf{S}_{n}, 1+x\right) \text { when } m \neq n \\
Z\left(\mathbf{S}_{n}^{\mathbf{S}_{2}}, 1+x\right) \text { when } m=n
\end{array}\right.
$$

4. k-colored graphs. We illustrate the general problem for $k=3$. Here we have, analogous to equations (3), (4) and (5), and with similar notation:

$$
\begin{aligned}
g_{m n t}(x) & =\sum_{q=0}^{m n t} g_{m n t, q} x^{q}, \\
g_{p}^{(3)}(x) & =\sum_{1 \leqq m \leqq n \leqq t} g_{m n t}(x), \\
g_{m n t}(x) & =Z\left(\Gamma_{1}\left(K_{m n t}\right), 1+x\right) .
\end{aligned}
$$

Thus $K_{m n t}$ is the complete tricolored graph with $m+n+t$ points $p_{1}, p_{2}, \cdots, p_{m}, q_{1}, q_{2}, \cdots, q_{n}, r_{1}, r_{2}, \cdots, r_{t}$ and all $m n+n t+t m$ lines of the 
form $p_{i} q_{\jmath}, q_{j} r_{k}$, and $r_{k} p_{i}$. Similarly $g_{m n t, q}$ is the number of spanning subgraphs of $K_{m n t}$ having $q$ lines, etc. We distinguish between three cases: (a) $m, n, t$ distinct, (b) $m=n \neq t$, and (c) $m=n=t$. These are illustrated in Figures $4(\mathrm{a}),(\mathrm{b})$, and (c).

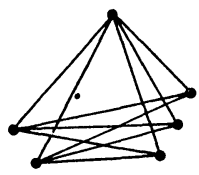

(a)

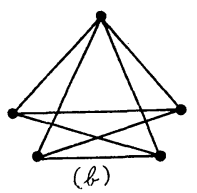

Fig. 4.

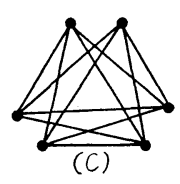

Only in case (a) have we obtained an algorithm for $Z\left(\Gamma_{1}\left(K_{m n t}\right)\right)$ in closed form. The result analogous to (9), derived in same manner, is as follows. Let $a_{1}$ to $a_{m}, b_{1}$ to $b_{n}$, and $c_{1}$ to $c_{t}$ be the indeterminates in $Z\left(\mathbf{S}_{m}\right), Z\left(\mathbf{S}_{n}\right)$, and $Z\left(\mathbf{S}_{t}\right)$ respectively. Let the indeterminates in $\left(\Gamma_{1}\left(K_{m n t}\right)\right)$ be $d_{1}, d_{2}, \cdots$. Let $A, B$, and $C$ denote arbitrary terms of $Z\left(\mathbf{S}_{m}\right), Z\left(\mathbf{S}_{n}\right)$, and $Z\left(\mathbf{S}_{t}\right)$ respectively. Then in this notation the left-hand member of equation (6) is $A \times B$ and the term of $Z\left(\Gamma_{1}\left(K_{m n t}\right)\right)$ obtained from $A, B$, and $C$ is,

$$
(A \times B)(B \times C)(C \times A),
$$

where each of the three factors in the expression (15) is a product of the indeterminates $d_{i}$ using equations (6) and (7). For example,

$$
Z\left(\Gamma_{1}\left(K_{123}\right)\right)=\frac{1}{12}\left(d_{1}^{11}+3 d_{2}^{3} d_{1}^{5}+2 d_{3}^{3} d_{1}^{2}+d_{2}^{4} d_{1}^{3}+3 d_{2}^{5} d_{1}+2 d_{3} d_{3} d_{2}\right)
$$

is the cycle index of the line group of the tricolored graph $K_{123}$ shown in Figure 4 (a).

Referring to Figure 4(b), one can find

$$
Z\left(\Gamma_{1}\left(K_{122}\right)\right)=\frac{1}{8}\left(d_{1}^{8}+2 d_{4}^{2}+4 d_{1}^{2} d_{2}^{3}+d_{2}^{4}\right) .
$$

The group $\Gamma_{1}\left(K_{122}\right)$ appears to be irreducible by any of the operations of direct product, cartesian product, composition, or exponentiation. However, it is abstractly isomorphic to $D_{4}$ and can be obtained from two copies of $\mathrm{D}_{4}$ defined on disjoint object sets $\{1,2,3,4\}$ and $\{5,6,7,8\}$ by the following operation.

Let the set $X$ be the union of the disjoint sets $X_{1}$ and $X_{2}$. Let $\mathbf{A}_{1}$ and $\mathbf{A}_{2}$ be permutation groups defined on $X_{1}$ and $X_{2}$ respectively, such that $h$ is an abstract isomorphism of $\mathbf{A}_{1}$ onto $\mathbf{A}_{2}$. Then the permutation group $\mathbf{A}_{1} \oplus_{h} \mathbf{A}_{2}$ can be defined as follows: The function $f$ from $X$ onto $X$ belongs to $\mathbf{A}_{1} \oplus_{h} \mathbf{A}_{2}$ if and only if there exist $\alpha_{1} \in \mathbf{A}_{2}$ and $\alpha_{2} \in \mathbf{A}_{2}$ 
with $\alpha_{2}=h \alpha_{1}$ such that $f(x)=\alpha_{1}(x)$ if $x \in X_{1}$ and $f(x)=\alpha_{2}(x)$ if $x \in X_{2}$. Clearly $\mathbf{A}=\mathbf{A}_{1} \bigoplus_{h} \mathbf{A}_{2}$ is abstractly isomorphic to $\mathbf{A}_{1}$.

Now let $D_{4,1}$ be the dihedral group of degree 4 generated by the permutations (1234) and (12)(34), let $D_{4,2}$ be generated by (5678) and (57)(6)(8), and let $h$ be the isomorphism between them which preserves respective generators. Then

$$
\Gamma_{1}^{\prime}\left(K_{122}\right)=\mathrm{D}_{4,1} \oplus_{h} \mathrm{D}_{4,2} .
$$

Finally, it is easy to see that $\Gamma_{1}\left(K_{222}\right)$ is abstractly isomorphic to $\mathbf{S}_{3}\left[\mathbf{S}_{2}\right]$ and that

$$
\begin{aligned}
Z\left(\Gamma_{1}\left(K_{222}\right)\right) & =\frac{1}{3 ! 2^{3}}\left[\left(c_{1}^{12}+3 c_{1}^{4} c_{2}^{4}+4 c_{2}^{6}\right)+3 \cdot 2\left(2 c_{1}^{3} c_{2}^{5}+2 c_{1}^{3}\right)+2 \cdot 2^{2}\left(c_{3}^{4}+c_{6}^{2}\right)\right] \\
& =\frac{1}{48}\left(c_{1}^{12}+3 c_{1}^{4} c_{2}^{4}+4 c_{2}^{6}+8 c_{3}^{4}+8 c_{6}^{2}+12 c_{1}^{2} c_{2}^{5}+12 c_{1}^{8}\right) .
\end{aligned}
$$

It is clear that the line group of $K_{n n n}$ is abstractly isomorphic to the automorphism group of $K_{n n n}$. Its complement $K_{n n n}^{\prime}$ consists of three disjoint copies of $K_{n}$, so that the group of $K_{n n n}$ is $S_{3}\left[S_{n}\right]$. But an explicit expression for $Z\left(\Gamma_{1}\left(K_{n n n}\right)\right)$ does not appear to be obvious. (For the particular case $n=3$, it can be shown that $\Gamma_{1}\left(K_{333}\right)$ is permutationally equivalent to $S_{3}^{S_{3}}$.) It does not appear that the operations considered here will suffice to enumerate even the tricolored graphs.

\section{Connected k-colored graphs. Let}

$$
g(x, y)=\sum_{p} g_{p}^{(k)}(x) y^{p}
$$

be the generating function for all (connected or not) k-colored graphs, and let $c(x, y)$ be that for the connected ones only. Then to find the number of connected $k$-colored graphs, we substitute into equation (33) of [2] to get

$$
1+g(x, y)=\exp \left(\sum_{n=1}^{\infty} \frac{1}{n} c\left(x^{n}, y^{n}\right)\right)
$$

or equivalently,

$$
\sum_{n=1}^{\infty} \frac{1}{n} c\left(x^{n}, y^{n}\right)=\sum_{n=1}^{\infty} \frac{(-1)^{n+1}}{n} g^{n}(x, y) .
$$

From either of these last two equations, the number of connected $k$-colored graphs of $p$ points can be computed recursively in terms of the total number of $k$-colored graphs. 
6. Balanced signed graphs. Signed graphs are obtained by assigning either a positive or a negative sign to each line of a graphs. It was indicated in [5] how one could enumerate all signed graphs by a variation in one of the formulas of [2]. The sign of a cycle is the product of the signs of its lines, and a signed graph is balanced if all its cycles are positive. The problem of enumerating balanced signed graphs was proposed in [5]. The result is derivable by an appropriate modification of the generating function for bicolored graphs.

It was shown in [5] that a signed graph is balanced if and only if its set of points can be partitioned into two disjoint subsets such that each positive line and no negative line joins two points in the same subset. In view of this characterization, called the "structure theorem for balance", on deleting all the positive lines of a balanced signed graph one obtains a bicolored graph. Let $G_{1}$ and $G_{2}$ be arbitrary graphs with $m$ and $n$ points respectively, $m \leq n$, and let $p=m+n$. Let $\Gamma\left(G_{1}\right)$ and $\Gamma\left(G_{2}\right)$ be the groups of $G_{1}$ and $G_{2}$ respectively. Let $b_{q}\left(G_{1}, G_{2}\right)$ be the number of nonisomorphic balanced signed graphs with q negative lines, whose positive lines generate the (disjoint) graphs $G_{1}$ and $G_{2}$ in accordance with the structure theorem for balance. Let

$$
b\left(G_{1}, G_{2}, x\right)=\sum_{q=0}^{m n} b_{q}\left(G_{1}, G_{2}\right) x^{q}
$$

be the desired configuration counting series. Then the figure counting series is $1+x$. For the figures are the $m n$ pairs of points $(c, d)$ where $c \in G_{1}$ and $d \in G_{2}$. The content of a figure $(c, d)$ is 0 if $c$ and $d$ are not joined by a negative line and is 1 if they are.

Analogously to the situation for bicolored graphs there are two possibilities. If $G_{1}$ and $G_{2}$ are not isomorphic, then the configuration group is $\Gamma\left(G_{1}\right) \times \Gamma\left(G_{2}\right)$. But if they are isomorphic, the configuration group is $\Gamma\left(G_{1}\right)^{\mathbf{s}_{2}}$. Hence an application of Pólya's Theorem yields

$$
b\left(G_{1}, G_{2}, x\right)= \begin{cases}Z\left(\Gamma\left(G_{1}\right) \times \Gamma\left(G_{2}\right), 1+x\right) & \text { when } G_{1} \not G_{2} \\ Z\left(\Gamma\left(G_{1}\right) \mathrm{S}_{2} 1,+x\right) & \text { when } G_{1} \cong G_{2}\end{cases}
$$

It is clear for the special case where $G_{1}$ and $G_{2}$ are the totally disconnected graphs of $m$ and $n$ points that $b\left(G_{1}, G_{2}, x\right)=g_{m n}(x), \Gamma\left(G_{1}\right)=\mathbf{S}_{m}$, and $\Gamma\left(G_{2}\right)=\mathbf{S}_{n}$. Thus the formula (17) is a generalization of that for bicolored graphs.

\section{REFERENCES}

1. G. A. Dirac, The structure of k-chromatic graphs, Fund. Math. 40 (1953), 42-55.

2. F. Harary, The number of linear, directed rooted and connected graphs. Trans. Amer.Math. Soc. 78 (1955), 445-463. 
3. - - On the number of dissimilar line-subgraphs of a given graph, Pacific. J. Math., 6 (1956), 57-64.

4. - - The number of dissimilar supergraphs of a linear graph, Pacific J. Math, 7 (1957), 903-911.

5. _ - On the notion of balance of a signed graph, Michigan Math. J. 2 (1953-54), 143-146.

6. G. Pôlya, Kombinatiorische Anzahlbestimmungen für Gruppen, Graphen, und chemishe, Verbindungen. Acta Math. 68 (1937), 145-254.

7. __ Sur les types des propositions composees, J. Symbolic Logic 5 (1940), 98-103.

8. D. Slepian, On the number of symmetry types of boolean functions of $n$ variables, Canadion J. Math. 5 (1953), 185-193.

University of Michigan and the Institute for Advanced Study 



\section{PACIFIC JOURNAL OF MATHEMATICS}

\section{EDITORS}

\section{David Gilbarg}

Stanford University

Stanford, California

\section{R. A. Beaumont}

University of Washington

Seattle 5, Washington

\author{
A. L. Whiteman
}

University of Southern California Los Angeles 7, California

E. G. Straus

University of California

Los Angeles 24, California

\section{ASSOCIATE EDITORS}

\author{
E. F. BECKENBACH \\ C. E. BURGESS \\ M. HALL \\ E. HEWITT
}
A. HORN
V. GANAPATHY IYER
R. D. JAMES
M. S. KNEBELMAN
L. NACHBIN
I. NIVEN
T. G. OSTROM
H. L. ROYDEN

M. M. SCHIFFER

G. SZEKERES

F. WOLF

K. YOSIDA

\section{SUPPORTING INSTITUTIONS}

\author{
UNIVERSITY OF BRITISH COLUMBIA \\ CALIFORNIA INSTITUTE OF TECHNOLOGY \\ UNIVERSITY OF CALIFORNIA \\ MONTANA STATE UNIVERSITY \\ UNIVERSITY OF NEVADA \\ OREGON STATE COLLEGE \\ UNIVERSITY OF OREGON \\ OSAKA UNIVERSITY \\ UNIVERSITY OF SOUTHERN CALIFORNIA
}

\author{
STANFORD UNIVERSITY \\ UNIVERSITY OF TOKYO \\ UNIVERSITY OF UTAH \\ WASHINGTON STATE COLLEGE \\ UNIVERSITY OF WASHINGTON \\ * * * * \\ AMERICAN MATHEMATICAL SOCIETY \\ CALIFORNIA RESEARCH CORPORATION \\ HUGHES AIRCRAFT COMPANY \\ THE RAMO-WOOLDRIDGE CORPORATION
}

Mathematical papers intended for publication in the Pacific Journal of Mathematics should be typewritten (double spaced), and the author should keep a complete copy. Manuscripts may be sent to any one of the four editors. All other communications to the editors should be addressed to the managing editor, E. G. Straus at the University of California, Los Angeles 24, California.

50 reprints per author of each article are furnished free of charge; additional copies may be obtained at cost in multiples of 50 .

The Pacific Journal of Mathematics is published quarterly, in March, June, September, and December. The price per volume (4 numbers) is $\$ 12.00$; single issues, $\$ 3.50$. Back numbers are available. Special price to individual faculty members of supporting institutions and to individual members of the American Mathematical Society: $\$ 4.00$ per volume; single issues, $\$ 1.25$.

Subscriptions, orders for back numbers, and changes of address should be sent to Pacific Journal of Mathematics, 2120 Oxford Street, Berkeley 4, California.

Printed at Kokusai Bunken Insatsusha (International Academic Printing Co., Ltd.), No. 6, 2-chome, Fujimi-cho, Chiyoda-ku, Tokyo, Japan.

PUBLISHED BY PACIFIC JOURNAL OF MATHEMATICS, A NON-PROFIT CORPORATION

The Supporting Institutions listed above contribute to the cost of publication of this Journal, but they are not owners or publishers and have no responsibility for its content or policies. 


\section{Pacific Journal of Mathematics}

\section{Vol. 8, No. $4 \quad$ June, 1958}

Richard Arens, The maximal ideals of certain functions algebras ........ 641

Glen Earl Baxter, An operator identity ........................... 649

Robert James Blattner, Automorphic group representations ........... 665

Steve Jerome Bryant, Isomorphism order for Abelian groups ............ 679

Charles W. Curtis, Modules whose annihilators are direct summands...... 685

Wilbur Eugene Deskins, On the radical of a group algebra ............ 693

Jacob Feldman, Equivalence and perpendicularity of Gaussian

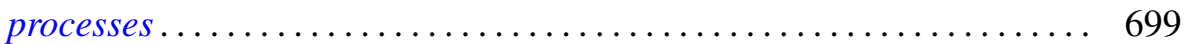

Marion K. Fort, Jr. and G. A. Hedlund, Minimal coverings of pairs by triples....................................... 709

I. S. Gál, On the theory of $(m, n)$-compact topological spaces ......... 721

David Gale and Oliver Gross, A note on polynomial and separable games........................................ 735

Frank Harary, On the number of bi-colored graphs ............... 743

Bruno Harris, Centralizers in Jordan algebras ................... 757

Martin Jurchescu, Modulus of a boundary component ............... 791

Hewitt Kenyon and A. P. Morse, Runs . . . . . . . . . . . . . . . . . . . . . . 811

Burnett C. Meyer and H. D. Sprinkle, Two nonseparable complete metric

spaces defined on $[0,1] \ldots \ldots \ldots \ldots \ldots \ldots \ldots \ldots \ldots \ldots \ldots . \ldots . \ldots . \ldots . \ldots 25$

M. S. Robertson, Cesàro partial sums of harmonic series expansions...... 829

John L. Selfridge and Ernst Gabor Straus, On the determination of numbers by their sums of a fixed order ........................ 847

Annette Sinclair, A general solution for a class of approximation

problems .................................

George Szekeres and Amnon Jakimovski, $(C, \infty)$ and $(H, \infty)$ methods of summation...................................... 867

Hale Trotter, Approximation of semi-groups of operators. ............. 887

L. E. Ward, A fixed point theorem for multi-valued functions ........... 921

Roy Edwin Wild, On the number of lattice points in $x^{t}+y^{t}=n^{t / 2} \ldots \ldots .929$ 